\title{
Comparison of Tn5397 from Clostridium difficile, Tn916 from Enterococcus faecalis and the CW459tet(M) element from Clostridium perfringens shows that they have similar conjugation regions but different insertion and excision modules
}

\author{
Adam P. Roberts, ${ }^{1}+$ Priscilla A. Johanesen, ${ }^{2}+$ Dena Lyras, ${ }^{2}$ Peter Mullany ${ }^{1}$ \\ and Julian I. Rood ${ }^{2}$
}

Author for correspondence: Peter Mullany. Tel: +44 207915 1223. Fax: +44 2079151127.

e-mail: p.mullany@eastman.ucl.ac.uk

1 Department of

Microbiology, Eastman Dental Institute for Oral Health Care Sciences, University College London, University of London, 256 Gray's Inn Road, London WC1X 8LD, UK

2 Bacterial Pathogenesis Research Group, Department of Microbiology, Monash University, Vic 3800, Australia

\begin{abstract}
Comparative analysis of the conjugative transposons Tn5397 from Clostridium difficile and Tn916 from Enterococcus faecalis, and the CW459tet(M) element from Clostridium perfringens, has revealed that these tetracycline-resistance elements are closely related. All three elements contain the tet(M) resistance gene and have sequence similarity throughout their central region. However, they have very different integration/excision modules. Instead of the int and xis genes that are found in Tn916, Tn5397 has a large resolvase gene, tndX. The C. perfringens element encodes the putative Int459 protein, which is a member of the integrase family of site-specific recombinases but is not closely related to Int from Tn916. Based on these studies it is concluded that the clostridial elements have a modular genetic organization and were derived independently from distinct mobile genetic elements.
\end{abstract}

Keywords: conjugative transposons, gene transfer, antibiotic resistance, mobile elements

\section{INTRODUCTION}

Conjugative transposons are mobile DNA elements that encode all the necessary functions for intracellular transposition and intercellular conjugation. They are found in a wide variety of both Gram-positive and Gram-negative bacteria and are important in the dissemination of antibiotic-resistance genes ( $\mathrm{Scott} \&$ Churchward, 1995). The first conjugative transposon identified, and the most extensively studied, is the $18 \mathrm{~kb}$ element Tn916 from Enterococcus faecalis strain DS16 (Franke \& Clewell, 1981). Tn916 and the closely related element Tn1545 from Streptococcus pneumoniae (Caillaud et al., 1987; Courvalin \& Carlier, 1987) form the basis of a family of conjugative transposons that have an extremely broad host range (Rice, 1998). All of

†These authors contributed equally to the work.

The GenBank accession numbers for the sequences in this paper are AF333235 (Tn5397) and AF329848 [part of CW459tet(M)]. the members of this family carry the tetracyclineminocycline-resistance gene, tet $(\mathrm{M})$ (Burdett, 1991); many also carry genes encoding resistance to other antimicrobial agents.

Conjugative transposition of members of the Tn916 family involves excision from the donor genome, circularization of the element, and then transfer to a new host and subsequent insertion into a new target site. Transposition requires the products of the transposonencoded int and xis genes, which encode Int, a sitespecific recombinase of the integrase family, and Xis, respectively (Poyart-Salmeron et al., 1990). Both Int and $\mathrm{Xis}$ are essential for excision but only Int is required for integration (Senghas et al., 1988; Poyart-Salmeron et al., 1990; Lu \& Churchward, 1995; Rudy et al., 1997; Marra \& Scott, 1999). Int-mediated excision of Tn916 is similar to the excision of $\lambda$ prophage DNA as it utilizes a mechanism that involves staggered cuts at the ends of the element, circularization, and subsequent transfer to a new host. However, the recombination sites of $\lambda$ are 
homologous, unlike those of Tn916 (Caparon \& Scott, 1989).

Members of the Tn916 family also encode their own conjugative transfer. Analysis of the complete DNA sequence of Tn916 reveals open reading frames (ORFs) that encode putative polypeptides with sequence similarity to proteins known to be involved in conjugation (Flannagan et al., 1994). For example, Orf18 has similarity to the anti-restriction protein Ard of plasmid Collb-P9 and Orf23 is related to the MbeA mobilization protein of plasmid ColE1. A functional oriT site is also present between orf21 and orf20 (Jaworski \& Clewell, 1995).

Work in our laboratories has concentrated on determining the molecular basis for tetracycline resistance in the anaerobic pathogens Clostridium difficile and Clostridium perfringens. In C. difficile, resistance is encoded by the conjugative transposon Tn5397, which mediates transfer between C. difficile strains and to and from Bacillus subtilis (Mullany et al., 1990). DNA hybridization analysis and partial DNA sequence analysis have shown that $\operatorname{Tn} 5397$ contains the tet $(\mathrm{M})$ gene and is closely related to Tn916. However, Tn5397 differs from Tn916 in that it contains a Group II intron inserted into orf14 (Mullany et al., 1996) and has different sequences at its ends (Wang et al., 2000a).

In C. perfringens, tetracycline resistance is the most common antimicrobial resistance phenotype (Lyras \& Rood, 1996). In most strains, this resistance is nontransferable; however, conjugative transfer of tetracycline resistance is not uncommon (Rood, 1983; Abraham et al., 1985). Transfer is always associated with large conjugative plasmids that are either identical to, or closely related to, the prototype R-plasmid, $\mathrm{pCW} 3$ (Abraham et al., 1985; Abraham \& Rood, 1985). pCW3 carries the well-characterized tetracycline-resistance determinant Tet $(\mathrm{P})$, which comprises two tetracyclineresistance genes, tet $A(\mathrm{P})$ and $\operatorname{tet} B(\mathrm{P})$ (Sloan et al., 1992). The $\operatorname{tet} A(\mathrm{P})$ gene encodes a $46 \mathrm{kD}$ a protein that mediates active efflux of tetracycline from the cell. The tet $B(\mathrm{P})$ gene encodes a putative $72 \cdot 6 \mathrm{kDa}$ protein that has significant similarity to Tet(M)-like tetracycline-resistance proteins.

Hybridization and PCR analysis of a large number of conjugative and non-conjugative tetracycline-resistant C. perfringens isolates has shown that they all carry the tet $A(\mathrm{P})$ gene. Most $(93 \%)$ of these isolates carry a second tetracycline-resistance gene, with $53 \%$ carrying a $\operatorname{tet} B(\mathrm{P})$ gene and $40 \%$ carrying a tet $(\mathrm{M})$-like gene (Lyras \& Rood, 1996). No isolates have been detected that carry both the tet $B(\mathrm{P})$ and the tet $(\mathrm{M})$-like genes. Conjugative transfer of the latter gene has not been demonstrated in C. perfringens.

In this paper, we compare the complete nucleotide sequences of Tn5397, Tn916 and the partial sequence of a tet $(\mathrm{M})$-like element from C. perfringens strain CW459. The results show that all three elements are closely related but have different excision modules. The comparison of these three elements provides valuable insights into the evolution and dissemination of conjugative transposons in Gram-positive bacteria.

\section{METHODS}

Bacterial strains and culture conditions. All Escherichia coli strains were derivatives of $\mathrm{DH} 5 \alpha$ (Life Technologies) or DH $5 \alpha \mathrm{MCR}^{\prime}$ (Gibco-BRL) and were grown at $37^{\circ} \mathrm{C}$ in $2 \times \mathrm{YT}$ (Sambrook et al., 1989) or LB medium (Sambrook et al., 1989) in the presence of $100 \mu \mathrm{g}$ ampicillin $\mathrm{ml}^{-1}, 10 \mu \mathrm{g}$ tetracycline $\mathrm{ml}^{-1}$ or $10 \mu \mathrm{g}$ minocycline $\mathrm{ml}^{-1}$. C. difficile strain 630 was grown in Brain Heart Infusion (BHI) broth or agar (Oxoid) at $37^{\circ} \mathrm{C}$ in an anaerobic chamber (Don Witley Scientific) in an atmosphere of $80 \% \mathrm{~N}_{2}, 10 \% \mathrm{H}_{2}$ and $10 \%$ $\mathrm{CO}_{2}$ in the presence of $10 \mu \mathrm{g}$ tetracycline $\mathrm{ml}^{-1}$ and $10 \mu \mathrm{g}$ erythromycin $\mathrm{ml}^{-1}$. C. perfringens strain CW459 was grown at $37^{\circ} \mathrm{C}$ in fluid thioglycollate medium (Difco) or trypticase peptone glucose broth (Rood et al., 1978b) or on nutrient (Rood, 1983) or BHI agar in an atmosphere of $80 \% \mathrm{~N}_{2}, 10 \%$ $\mathrm{H}_{2}$ and $10 \% \mathrm{CO}_{2}$ in an anaerobic jar (Oxoid). Tetracycline $\left(5 \mu \mathrm{g} \mathrm{ml}^{-1}\right)$ was added when appropriate.

Molecular techniques. Plasmid DNA was isolated from E. coli using the Qiagen Miniprep kit or the High Pure Plasmid Isolation Kit (Boehringer Mannheim) in accordance with the manufacturers' instructions or using a modified small-scale mini alkaline/lysis/PEG precipitation procedure (Applied Biosystems). Genomic DNA was prepared from C. difficile by using the Puregene Gram-positive genomic DNA isolation kit (Flowgen) and from C. perfringens using dye-buoyant gradient ultracentrifugation at $260000 \mathrm{~g}$ for $20 \mathrm{~h}$ at $20^{\circ} \mathrm{C}$ (Abraham \& Rood, 1985).

A genomic library of C. perfringens strain CW459 (Rood et al., 1978a) was constructed by digesting purified chromosomal DNA with PstI and ligating it to PstI-digested pSU39 (Bartolome et al., 1991). After overnight ligation at $14{ }^{\circ} \mathrm{C}$, the DNA was introduced into rubidium chloride competent $E$. coli DH5 $\alpha$ cells (Sambrook et al., 1989). The library was screened on media containing tetracycline $\left(10 \mu \mathrm{g} \mathrm{ml}^{-1}\right)$. Recombinant clones that were tetracycline resistant were screened for minocycline resistance $\left(10 \mu \mathrm{g} \mathrm{ml}^{-1}\right)$. The recombinant plasmid pJIR1470 was found to confer resistance to both tetracycline and minocycline.

DNA sequencing and analysis. DNA sequencing was carried out using the ABI Prism Big Dye Terminator Cycle Sequencing Ready Reaction Kit on an Applied Biosystems Perkin Elmer 310 Genetic Analyser or an Applied Biosystems 373 DNA Sequencer. DNA sequence analysis was carried out using the DNASIS (Hitachi), NCBI tools (http://www.ncbi.nlm.nih. gov/), European Bioinformatics Institute tools (http:// www.ebi.ac.uk/), Seqed (Applied Biosystems), Sequencer 3.0 Software (Gene Codes Corporation) and Gene Jockey (Biosoft) programs.

Dot blot hybridization analysis. Plasmid DNA from E. coli and chromosomal DNA from C. perfringens and C. difficile were applied to Hybond-N + nylon membranes (Amersham) without prior treatment (Sambrook et al., 1989) using a dot blot apparatus (Minifold SRC 96; Schleicher \& Schuell). Analysis of dot blots was performed using the DIG DNA Labelling and Detection Kit (Boehringer Mannheim) in accordance with the manufacturer's instructions. Hybridization was carried out at $65^{\circ} \mathrm{C}$ in $5 \times \operatorname{SSC}(0.75 \mathrm{M} \mathrm{NaCl}$, $0.075 \mathrm{M}$ sodium citrate $\mathrm{pH} 7 \cdot 0$ ) with subsequent washes of $2 \times 15 \mathrm{~min}$ at $65^{\circ} \mathrm{C}$ in $0 \cdot 1 \times \mathrm{SSC}, 0 \cdot 1 \%$ (w/v) SDS. Digoxigenin-11-dUTP-labelled probes were amplified by PCR from Tn916 template DNA and spanned the length of the Tn916 transposon (Fig. 1). 


\section{RESULTS AND DISCUSSION}

\section{The Tet(M) determinant from $C$. perfringens strain CW459 is located on a Tn916-like element}

Previous studies have identified C. perfringens strains that carry tet $(\mathrm{M})$ genes (Lyras \& Rood, 1996). The tet $(\mathrm{M})$ determinant from one such strain, CW459, was cloned into the plasmid vector pSU39 to generate pJIR1470. Restriction endonuclease digestion showed that this plasmid contained a $28 \cdot 1 \mathrm{~kb}$ Pst I insert and DNA hybridization confirmed that it contained the tet $(\mathrm{M})$ gene (data not shown). To see if pJIR1470 carried homologues of other Tn916-encoded genes, the plasmid was hybridized with a series of separate PCR-derived DIG-labelled probes that together encompassed most of Tn916 (Fig. 1). C. difficile strain 630, which carries Tn5397, C. perfringens strain JIR4229 (Awad \& Rood, 1997), which carries a chromosomal copy of Tn916, and pAM120, an E. coli plasmid carrying Tn916, were included as positive controls. The results (Fig. 1) showed that pJIR1470 hybridized to each of the Tn916-derived probes, suggesting that a complete Tn916-like element was present in this plasmid and consequently in strain CW459. However, since this strain is non-conjugative (Rood et al., 1978a), and no evidence for the transposition of its tet $(\mathrm{M})$ gene has been obtained, this region was designated the CW459tet $(\mathrm{M})$ element but not given a formal transposon designation. As expected, C. difficile strain 630 also hybridized to these probes (Hächler et al., 1987).

\section{Comparison of the overall genetic organization of Tn5397, Tn916 and CW459tet(M)}

Previous studies involved the sequence analysis of the first $328 \mathrm{bp}$ of the left end and the last $2442 \mathrm{bp}$ of the right end of Tn5397 (Wang et al., 2000a). The remaining sequence of $\operatorname{Tn} 5397$ was determined on both strands by primer walking on the plasmids pPPM5.3 and pPPM1.6 (Mullany et al., 1996). Gaps in the sequence were filled by designing PCR primers based on the Tn916 sequence (GenBank accession no. U09422) and the unpublished C. difficile sequence (http://www.sanger.ac.uk/Projects/C_difficile/). To avoid PCR-induced errors, each PCR product was amplified and sequenced at least three times. The results showed that Tn5397 was 20658 bp in length (GenBank accession no. AF333235) and had 21 potential ORFs that had a suitably spaced ribosome-binding site upstream of the putative start codon. The ORF within the Group II intron (Mullany et al., 1996) and tndX (Wang et al., 2000a; Wang \& Mullany, 2000) have been described previously. Seventeen of the ORFs were very similar to corresponding ORFs from Tn916 (Flannagan et al., 1994) and were labelled accordingly (Fig. 2).

The CW459tet(M) element was sequenced on both strands by primer walking on a pJIR1470 template. An $8691 \mathrm{bp}$ region including the tet $(\mathrm{M})$ gene and the right end of the element was sequenced (GenBank accession no. AF329848). The $\mathrm{G}+\mathrm{C}$ content of this region was
$35.9 \mathrm{~mol} \%$ compared to $38.3 \mathrm{~mol} \%$ for Tn5397 and $38.8 \mathrm{~mol} \%$ for Tn916. Since CW459tet(M) is not a functional mobile element, the left end of the element was not completely sequenced. The overall structure of both clostridial elements is very similar to that of Tn916 (Fig. 2) although there are numerous insertions, deletions and rearrangements, both within ORFs and in the intergenic regions. This finding also applies to the incomplete single-stranded sequence that was obtained from the left end of the CW459tet $(\mathrm{M})$ element (data not shown). Nine of the ten sequenced CW459tet(M) ORFs were closely related to those of $\operatorname{Tn} 916$ and were named accordingly (Fig. 2). A comparison of each of these putative protein products is shown in Table 1.

\section{Tn5397, CW459tet(M) and Tn916 have different excision/insertion modules}

The most notable differences between the three elements occurred at the extreme right end, where both clostridial elements diverged from Tn916 and from each other (Fig. 2). In these elements, divergence from Tn916 occurs within orf5, suggesting that this region is a recombinational hotspot. Tn5397 only has the $3^{\prime}$ end of orf5 but is still capable of conjugative transfer, providing evidence that orf5 is not essential for the transfer of this element.

In Tn916, the int and xis genes, the products of which are essential for excision/insertion, are located to the right of $\operatorname{orf5}$ (Fig. 2). In the clostridial elements, these genes have been replaced with other genes that encode different site-specific recombinases. Database searches with the putative protein encoded at the right end of the CW459tet $(\mathrm{M})$ element revealed $21-28 \%$ identity to several bacteriophage or transposon-encoded integrases from Gram-positive bacteria (GenBank accession nos AAA 85500, AAC48867, A69774 and C55205) and $17 \cdot 5 \%$ identity to Int from Tn916. Therefore, the CW459derived ORF was designated int459 (integrase CW459). A comparison of Int 459 with other members of the integrase family showed that the conserved integrase motif (Abremski \& Hoess, 1992) was present in the putative Int 459 protein. This protein may have played a role in the transposition and introduction of the CW459tet $(\mathrm{M})$ element into CW459. However, since conjugative transfer of the CW459tet(M) element is not observed, it appears that either this protein is not produced, is not functional, or is not sufficient on its own for transposition. As observed for the transposition of Tn916 (Jaworski et al., 1996; Marra \& Scott, 1999), the excision, and therefore transposition, of the CW459tet $(\mathrm{M})$ element may require additional proteins, such as an excisionase, which are not present in this element.

An additional $170 \mathrm{bp}$ incomplete ORF was identified at the right of the sequenced CW459tet $(\mathrm{M})$ region and was designated gmp because the deduced protein product had $70 \%$ identity over 47 amino acids to a GMP synthetase protein from B. subtilis (Mantsala \& Zalkin, 1992). Since this enzyme is a housekeeping protein we 
(a)
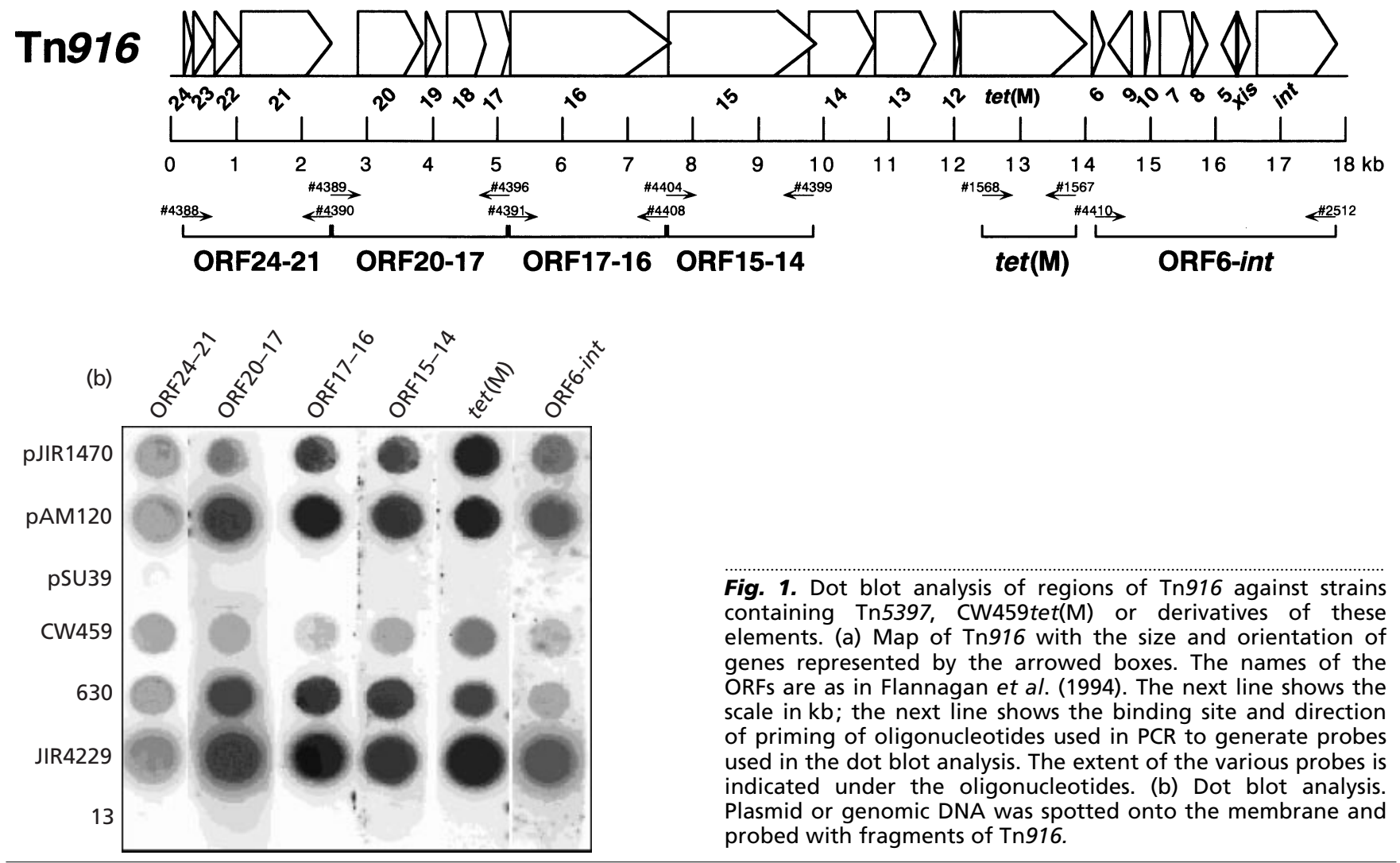

$\operatorname{Tn} 5397$

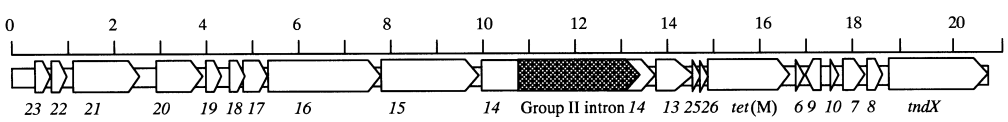

$\operatorname{Tn} 916$

$\underset{242322}{0}$

Fig. 2. Genetic organization of Tn5397 and CW459tet(M) compared to Tn916. The top line for all three elements indicates the size in $\mathrm{kb}$. Underneath this is the genetic structure of the elements. Arrowed boxes represent the ORFs, with the proposed direction of transcription shown by the arrow. The ORFs are labelled underneath. In

CW459tet(M)

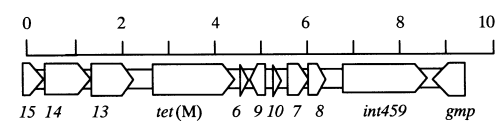
Tn5397, the Group II intron is shaded. In Tn916, a black arrowhead depicts the oriT. Only the sequenced area of CW459tet(M) is shown.

concluded that it was outside the CW459tet(M) element. Therefore, the right terminus of the element appeared to be located within the $67 \mathrm{bp}$ region between int459 and gmp (Fig. 2). Although this region contained several repeat sequences, it did not have any similarity to the ends of Tn916, Tn5397 or any other known conjugative transposons.

Downstream of orf8 in Tn5397 (Fig. 2) is the $t$ ndX gene, which encodes a $61.5 \mathrm{kDa}$ protein, TndX (Wang et al., 2000a), that has $37 \%$ identity to TnpX, a large resolvase from the C. perfringens transposon Tn4451 (Bannam et al., 1995) and the C. difficile transposon Tn4453a (Lyras
\& Rood, 2000). Functional analysis has shown that TndX is involved in the excision and integration of Tn5397 in C. difficile. The tndX gene ends 10 bp before the right terminus of Tn5397 (Wang et al., 2000a ; Wang \& Mullany, 2000).

\section{The Tn5397 and CW459tet(M) elements do not have the tet(M) leader peptide orf12}

Just upstream of the tet $(\mathrm{M})$ gene in $\operatorname{Tn} 916$ there is a small ORF, orf12, which encodes a tet $(\mathrm{M})$ leader peptide that is involved in the regulation of tet $(\mathrm{M})$ expression by 
Table 1. Comparative analysis of the Orf14-Orf5 region of the CW459tet(M) element, Tn916 and Tn5397

Percentage identity is shown.

\begin{tabular}{|c|c|c|}
\hline & $\operatorname{Tn} 916$ & CW459 \\
\hline \multicolumn{3}{|l|}{ Orf14 } \\
\hline Tn916 & $100 \cdot 0$ & \\
\hline CW459 & $71 \cdot 5$ & $100 \cdot 0$ \\
\hline Tn5397 & $87 \cdot 4$ & $66 \cdot 3$ \\
\hline \multicolumn{3}{|l|}{ Orf13 } \\
\hline $\operatorname{Tn} 916$ & $100 \cdot 0$ & \\
\hline CW459 & $52 \cdot 1$ & $100 \cdot 0$ \\
\hline Tn5397 & $87 \cdot 4$ & $52 \cdot 7$ \\
\hline \multicolumn{3}{|l|}{$\operatorname{Tet}(\mathbf{M})$} \\
\hline $\operatorname{Tn} 916$ & $100 \cdot 0$ & \\
\hline CW459 & $99 \cdot 7$ & $100 \cdot 0$ \\
\hline Tn5397 & $90 \cdot 5$ & $90 \cdot 5$ \\
\hline \multicolumn{3}{|l|}{ Orf6 } \\
\hline $\operatorname{Tn} 916$ & $100 \cdot 0$ & \\
\hline CW459 & $100 \cdot 0$ & $100 \cdot 0$ \\
\hline $\operatorname{Tn} 5397$ & $87 \cdot 1$ & $87 \cdot 1$ \\
\hline \multicolumn{3}{|l|}{ Orf9 } \\
\hline Tn916 & $100 \cdot 0$ & \\
\hline CW459 & $99 \cdot 1$ & $100 \cdot 0$ \\
\hline $\operatorname{Tn} 5397$ & $83 \cdot 2$ & $84 \cdot 0$ \\
\hline \multicolumn{3}{|l|}{ Orf10* } \\
\hline Tn916 & $100 \cdot 0$ & \\
\hline CW459 & $43 \cdot 4$ & $100 \cdot 0$ \\
\hline $\operatorname{Tn} 5397$ & $47 \cdot 8$ & $39 \cdot 1$ \\
\hline \multicolumn{3}{|l|}{ Orf7 } \\
\hline Tn916 & $100 \cdot 0$ & \\
\hline CW459 & $68 \cdot 6$ & $100 \cdot 0$ \\
\hline Tn5397 & $85 \cdot 8$ & $63 \cdot 8$ \\
\hline \multicolumn{3}{|l|}{ Orf8 } \\
\hline $\operatorname{Tn} 916$ & $100 \cdot 0$ & \\
\hline CW459 & $78 \cdot 9$ & $100 \cdot 0$ \\
\hline Tn5397 & $85 \cdot 5$ & $69 \cdot 7$ \\
\hline \multicolumn{3}{|l|}{$\Delta$ Orf5 $†$} \\
\hline $\operatorname{Tn} 916$ & $100 \cdot 0$ & \\
\hline CW459 & $53 \cdot 2$ & $100 \cdot 0$ \\
\hline Tn5397 & $70 \cdot 9$ & $59 \cdot 6$ \\
\hline
\end{tabular}

* Over 23 amino acids

† Over 62 amino acids.

a transcriptional attenuation mechanism ( $\mathrm{Su}$ et al., 1992; Celli \& Trieu-Cuot, 1998). In both Tn5397 and the CW459tet(M) element there has been a deletion of all or part of orf12. In Tn5397, the $88 \mathrm{bp}$ deletion is associated with a CCCAGT direct repeat (Fig. 3). In the $\mathrm{CW} 459$ tet $(\mathrm{M})$ element, there is a deletion of $155 \mathrm{bp}$, which is associated with a CCTTTT direct repeat (Fig. 3). Therefore, both deletions appear to have arisen by homologous recombination. CW459 expresses tetracycline resistance constitutively (J. Rood, unpublished results), but also carries a second tetracycline-resistance gene, tet $A(P)$. Strain 630 is inducible but the mechanism of induction of tetracycline resistance is not known (A. Roberts \& P. Mullany, unpublished results). However, although orf12 is partially deleted, this region still contains an inverted repeat $\left(\Delta G=-61.7 \mathrm{~kJ} \mathrm{~mol}^{-1}\right)$ that has similarity to the repeats which in Tn916 form the upstream transcriptional terminator.

\section{The Tet(M) proteins from Tn916 and the CW459tet(M) element are more closely related to each other than to Tet(M) from Tn5397}

The deduced amino acid sequence of the Tet $(M)$ protein from the CW459tet $(\mathrm{M})$ element was almost identical to the Tet $(\mathrm{M})$ protein from $\mathrm{Tn} 916$ (Su et al., 1992; Flannagan et al., 1994) but was less closely related to the Tet $(\mathrm{M})$ protein from Tn5397 (Table 1). Comparative analysis was also performed with other $\operatorname{Tet}(\mathrm{M})$ proteins from a variety of bacterial genera. A high degree of sequence identity (91-99\%) was observed (Fig. 4). Tet(M) from Tn5397 had slightly higher identity (93\%) to the Tet(M) protein from Tn1545 (Martin et al., 1986) than to the Tet(M) protein from CW459 or Tn916 (Table 1). These results suggest that the acquisition of the CW459 resistance determinant from a precursor carrying a Tn916-like tet $(\mathrm{M})$ gene was a relatively recent event and that the tet(M) genes in CW459 and Tn5397 were not derived from each other but originated from different donors at different times.

\section{The regions between tet(M) and the recombinase genes are similar but not identical to the equivalent region of $\operatorname{Tn} 916$}

The regions between tet $(\mathrm{M})$ and the recombinase genes are closely related in all three elements (Table 1). However, the CW459tet(M) ORFs are more closely related to those of Tn916 than to Tn5397 (Table 1). Celli \& Trieu-Cuot (1998) showed that orfs 7, 8 and 9 encoded putative regulatory proteins. Homologues of these ORFs are present in similar size and arrangement in the clostridial elements (Table 1). The proposed regulatory region in $\mathrm{Tn} 916$ includes the palindromes

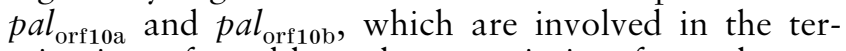
mination of readthrough transcription from the upstream tet $(\mathrm{M})$ promoter. Similar but not identical palindromes are found in both clostridial elements.

In both Tn5397 and the CW459tet(M) element there was an intergenic region after orf8, followed by the remnants of orf5. In both elements, the N-terminal encoding region of orf5 is missing. In $\mathrm{Tn} 916$, the orf8-orf5 intergenic region contains the promoter region for the xis and int genes (Celli \& Trieu-Cuot, 1998). The intergenic region in the CW459tet(M) element was similar in size and nucleotide sequence identity $(79 \%)$ to the equivalent region of $\operatorname{Tn} 916$. The xis-int promoter region was present but there were two single base changes. Two regions of dyad symmetry, which have some similarity to the imperfect inverted repeats $p a l_{\text {orf } 8 \text { a }}$ 


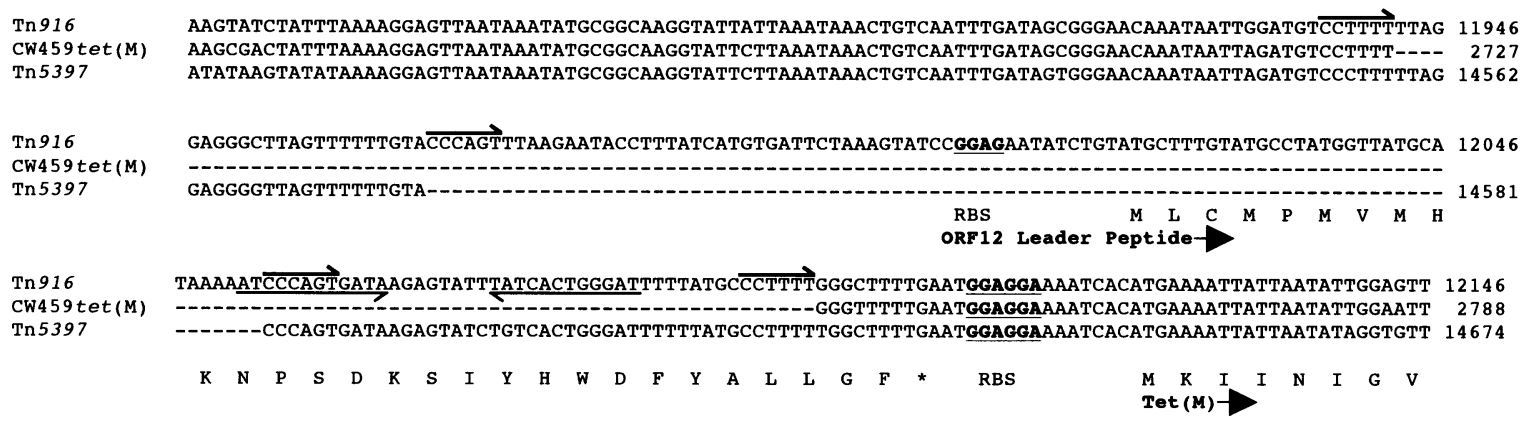

Fig. 3. Comparative DNA sequence analysis of the region just upstream of tet(M) in Tn5397, Tn916 and CW459tet(M). The deduced amino acid sequence of the Orf12 leader and Tet(M) of Tn916 is shown in the single letter code underneath the nucleotide sequence. Ribosome-binding sites are underlined and shown in bold. Inverted repeats within orf12 are underlined or overlined with half arrows. Direct repeats (CCTTTT) associated with the 155 bp deletion region upstream of the CW459tet(M) tet gene are overlined by half arrows. Direct repeats (CCCAGT) associated with the 88 bp deletion in Tn5397 are overlined by half arrows.

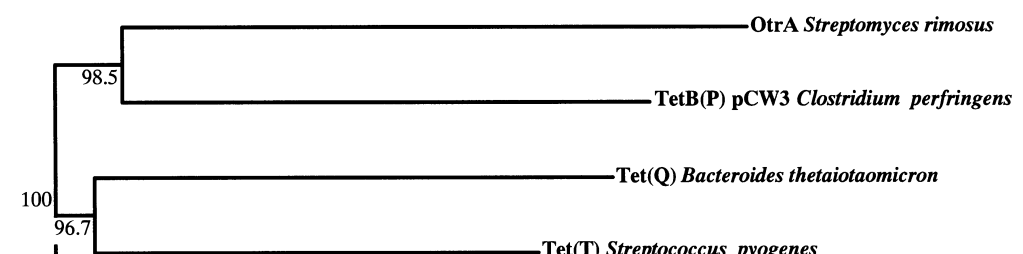

Fig. 4. Phylogenetic relationship between ribosomal protection proteins. The relationship of ribosomal protection proteins was estimated using the neighbour-joining distance procedure (Thompson et al., 1994). The alignment was constructed using CLUSTAL W (Thompson et al., 1994). The reliability of the branching was assessed using bootstrapping analysis (1000 replications). Statistical significant support $(<70 \%)$ for a branch is indicated at the node as a percentage. The branch lengths are scaled in proportion to the extent of the change per position as indicated by the scale bar. No correction for multiple substitution was made. The tree showing the evolutionary relationships between these proteins was constructed using TreeView (Rhoderic D. M. Page). The phylogenetic tree was constructed from amino acid sequences from the following proteins: Tet(S) from Lactococcus lactis (accession no. CAA63528), Tet(O) from Campylobacter jejuni (accession no. AAA23033), Tet(W) from Butyrivibrio fibrisolvens (accession no. CAA10975), Tet(T) from Streptococcus pyogenes (accession no. AAF01499), TetB $(P)$ from $C$. perfringens (accession no. AAA20117) and OtrA from Streptomyces rimosus (accession no. S18572). $\operatorname{Tet} B(P)$ and OtrA were used as outgroups as they are the most distantly related ribosomal protection proteins.

and pal $_{\text {orfsb }}$ from Tn916, were present. These repeats are believed to be involved in transcriptional termination from the upstream orf7 promoter (Celli \& Trieu-Cuot, 1998). In contrast, the smaller orf8-orf5 intergenic region of $\operatorname{Tn} 5397$ has sequence identity $(74 \%)$ to only part of the equivalent Tn916 region. In Tn5397, a major deletion has occurred at the beginning of this region. This deletion effectively removes the promoter area of 
the missing xis and int genes and the pal ${ }_{\text {orfs }}$ repeats. To the right of the remainder of orf5 the sequence of the clostridial elements diverges dramatically as already discussed.

\section{Tn5397 contains homologues of the Tn916 conjugation genes}

As previously described, the $180 \mathrm{bp}$ at the left end of Tn5397 are not related to Tn916 (Wang et al., 2000a). The first seven nucleotides of orf 24 are also missing. Following this region, the two transposons are very closely related (Fig. 2) although several insertions and deletions are present in Tn5397. The deduced amino acid sequences of Orfs $23-16$ have $90-100 \%$ identity to their Tn916 homologues; Orf15 has $80 \%$ identity. Following orf 21 is a 392 bp intergenic region which contains a sequence that is homologous to the functional oriT region of Tn916 (Jaworski \& Clewell, 1995). Within the oriT sites of Tn916 and the F plasmid there is a conserved nick site, $5^{\prime}$-TGGTGTGG- $3^{\prime}$. This site is identical in Tn5397.

Preliminary sequence analysis of the CW459tet(M) element indicated that sequences with similarity to much of the region encompassing the conjugation genes of Tn916 appear to be present. However, its exact genetic organization and precise relationship to the equivalent region in Tn916 remain to be determined. The conserved oriT nick site is present.

The first ORF of the double-stranded sequenced region of the CW459tet $(\mathrm{M})$ element, orf15, was only partially sequenced. However, the amino acid sequence of the deduced protein had only $21 \%$ identity to a corresponding 288-amino-acid region of Orf15 from Tn916 (Flannagan et al., 1994) and $23.9 \%$ identity to a corresponding region of Orf15 from Tn5397. By comparison with Tn916, Orf15 from both Tn5397 and the $\mathrm{CW} 459$ tet $(\mathrm{M})$ element contained C-terminal truncations of 25 and 57 amino acids, respectively. In Tn5397, the next ORF, orf14, is disrupted by a Group II intron (Mullany et al., 1996). Neither the CW459tet(M) element nor Tn916 contains a Group II intron in orf14 (Fig. 2). Comparative analysis of the deduced amino acid sequences showed that Orf14 (minus the Group II intron) from Tn5397 is most closely related to Orf14 from Tn916.

\section{Evolution of Tn5397 and the CW459tet(M) element}

Both Tn5397 and the CW459tet(M) element appear to consist of four distinct modules that may have originated from separate mobile genetic elements. The first module is common although not identical and consists of the Tn916-like conjugation region (orf23-orf13), which is present in Tn5397 and most probably in the $\mathrm{CW} 459$ tet $(\mathrm{M})$ element. The resistance determinant comprises the second module, with the tet $(\mathrm{M})$ genes of Tn916 and CW459 being very closely related and distinct from that of Tn5397, which is more closely related to the tet $(\mathrm{M})$ gene from Tn1545 (Fig. 4). The third module comprises the putative regulatory region (orf12-orf8). The regulatory and resistance modules may overlap, as orf12 is involved in sensing tetracycline and consequently regulating tet $(\mathrm{M})$ expression. The fourth major module is responsible for the excision and insertion of the transposons or their precursors and consists of xis and int in Tn916, int459 in CW459tet(M) and tndX in Tn5397. Therefore, the three elements have different excision and integration systems.

Tn916-like sequences have also been found in other mobile genetic elements. The tet $(\mathrm{M})$ genes of the large conjugative plasmids of Neisseria gonorrhoeae and Neisseria meningitidis are associated with Tn916-like sequences that encompass regions upstream and downstream of the resistance determinant (Swartley et al., 1993). Other studies involving the non-conjugative multiple antibiotic resistance plasmid pK214 from $L$. lactis, which carries resistance determinants for streptomycin, tetracycline and chloramphenicol, have revealed that the tetracycline-resistance gene, $\operatorname{tet}(\mathrm{S})$, is linked to sequences with similarity to orf7, orf 9 and orf6, which have a similar genetic organization to that observed in Tn916. This Tn916-like region is linked to distinct gene regions from three other bacterial species as well as to numerous insertion sequences (Perreten et al., 1997; Teuber et al., 1999). Finally, the integrase from a lysogenic Mycoplasma arthriditis bacteriophage has greater similarity to Int from Tn916 than to any other integrase (Voelker \& Dybvig, 1998), suggesting a common evolutionary origin.

There are several hypotheses that may explain the evolution of the non-functional CW459tet(M) element. Firstly, it is possible that a modular conjugative transposon may have been initially formed by recombination events between different mobile genetic elements in another bacterium and then subsequently transferred to C. perfringens. Upon insertion into the chromosome, deletion or rearrangement events may have occurred which resulted in the loss of mobility of the transposon. Similar events, resulting in deletions of sections of Tn916, have been observed in Neisseria (Swartley et al., 1993). Altered Tn916-like elements, which are no longer capable of conjugative transfer, have also been detected in the non-conjugative plasmid pKQ1, from Enterococcus faecium (Fletcher \& Daneo-Moore, 1992). Secondly, a composite element may have been formed in $C$. perfringens strain CW459 by a recombination event between a previously acquired, ancestral Tn916 or Tn916-like transposon and a second mobile genetic element, which carried an Int459-based excision/ integration system. Alternatively, the recombination event may have involved a resident element containing int459 and an incoming Tn916-like transposon. Note that the conjugative transposition of Tn916 into the chromosome of C. perfringens from both E. coli and Enterococcus faecalis has been observed (Kaufmann et al., 1996). Tn916 has also been shown to transfer into $C$. difficile from B. subtilis (Mullany et al., 1991) and natural isolates of $C$. difficile that carry Tn916 have recently been identified (Wang et al., 2000b). 
Although direct transfer of genetic information between C. perfringens and C. difficile has not been demonstrated, closely related transposons have been detected in both species, indicating that genetic exchange whether directly, or indirectly through another bacterial host, can occur (Lyras et al., 1998). The TndX protein, which is involved in the excision and integration of Tn5397, is more closely related to the TnpX resolvase that is responsible for the movement of the C. perfringens transposon Tn4451 and the closely related C. difficile Tn4453 transposons than to any other resolvase (Wang et al., 2000a; Lyras \& Rood, 2000). It has been proposed that one of these elements may have been involved in recombination events with an ancestral Tn916-like element that resulted in the formation of Tn5397 (Wang et al., 2000a).

\section{ACKNOWLEDGEMENTS}

A.P.R. was the recipient of a BBSRC studentship. Work in the London laboratory was funded by the Wellcome Trust. Work in the Monash laboratory was supported by grants from the Australian National Health and Medical Research Council.

\section{REFERENCES}

Abraham, L. J. \& Rood, J. I. (1985). Cloning and analysis of the Clostridium perfringens tetracycline resistance plasmid, pCW3. Plasmid 13, 155-162.

Abraham, L. J., Wales, A. J. \& Rood, J. I. (1985). Worldwide distribution of the conjugative Clostridium perfringens tetracycline resistance plasmid, pCW3. Plasmid 14, 37-46.

Abremski, K. E. \& Hoess, R. H. (1992). Evidence for a second conserved arginine residue in the integrase family of recombination proteins. Protein Eng 5, 87-91.

Awad, M. M. \& Rood, J. I. (1997). Isolation of alpha-toxin, thetatoxin and kappa-toxin mutants of Clostridium perfringens by Tn916 mutagenesis. Microb Pathog 22, 275-284.

Bannam, T. L., Crellin, P. K. \& Rood, J. I. (1995). Molecular genetics of the chloramphenicol-resistance transposon Tn4451 from Clostridium perfringens: the TnpX site-specific recombinase excises a circular transposon molecule. Mol Microbiol 16, 535-551.

Bartolome, B., Jubete, Y., Martinez, E. \& de la Cruz, F. (1991). Construction and properties of pACYC184-derived cloning vectors compatible with pBR322 and its derivatives. Gene 102, 75-78.

Burdett, V. (1991). Purification and characterization of Tet(M), a protein that renders ribosomes resistant to tetracycline. $\mathrm{J} \mathrm{Biol}$ Chem 15, 2872-2877.

Caillaud, F., Carlier, C. \& Courvalin, P. (1987). Physical analysis of the conjugative shuttle transposon Tn1545. Plasmid 1, 58-60.

Caparon, M. G. \& Scott, J. R. (1989). Excision and insertion of the conjugative transposon Tn916 involves a novel recombination mechanism. Cell 22, 1027-1034.

Celli, J. \& Trieu-Cuot, P. (1998). Circularisation of Tn916 is required for expression of the transposon-encoded transfer functions: characterisation of long tetracycline-inducible transcripts reading through the attachment site. Mol Microbiol 28, 103-117.

Courvalin, P. \& Carlier, C. (1987). Tn1545: a conjugative shuttle transposon. Mol Gen Genet 206, 259-264.
Flannagan, S. E., Zitzow, L. A., Su, Y. A. \& Clewell, D. B. (1994). Nucleotide sequence of the $18 \mathrm{~kb}$ conjugative transposon Tn916 from Enterococcus faecalis. Plasmid 32, 350-354.

Fletcher, H. M. \& Daneo-Moore, L. (1992). A truncated Tn916-like element in a clinical isolate of Enterococcus faecium. Plasmid 27, $155-160$.

Franke, A. E. \& Clewell, D. B. (1981). Evidence for a chromosome borne resistance transposon (Tn916) in Streptococcus faecalis that is capable of 'conjugal' transfer in the absence of a plasmid. J Bacteriol 145, 494-502.

Hächler, H., Kayser, F. H. \& Berger-Bächi, B. (1987). Homology of a transferable tetracycline resistance determinant of Clostridium difficile with Streptococcus (Enterococcus) faecalis transposon Tn916. Antimicrob Agents Chemother 31, 1033-1038.

Jaworski, D. D. \& Clewell, D. B. (1995). A functional origin of transfer (oriT) on the conjugative transposon Tn916. J Bacteriol 177, 6644-6651.

Jaworski, D. D., Flannagan, S. E. \& Clewell, D. B. (1996). Analyses of traA, int-Tn, and xis-Tn mutations in the conjugative transposon Tn916 in Enterococcus faecalis. Plasmid 36, 201-208.

Kaufmann, P., Lehmann, Y. \& Meile, L. (1996). Conjugative transposition of Tn916 from Enterococcus faecalis and Escherichia coli into Clostridium perfringens. Syst Appl Microbiol 19, 35-39.

Lu, F. \& Churchward, G. (1995). Tn916 target DNA sequences bind the C-terminal domain of integrase protein with different affinities that correlate with transposon insertion frequency. $J$ Bacteriol 177, 1938-1946.

Lyras, D. \& Rood, J. I. (1996). Genetic organization and distribution of tetracycline resistance determinants in Clostridium perfringens. Antimicrob Agents Chemother 40, 2500-2504.

Lyras, D. \& Rood, J. I. (2000). Transposition of Tn4451 and Tn4453 involves a circular intermediate that forms a promoter for the large resolvase, tnpX. Mol Microbiol 38, 588-601.

Lyras, D., Storie, C., Huggins, A. S., Crellin, P. K., Bannam, T. L. \& Rood, J. I. (1998). Chloramphenicol resistance in Clostridium difficile is encoded on Tn4453 transposons that are closely related to Tn4451 from Clostridium perfringens. Antimicrob Agents Chemother 23, 784-786.

Mantsala, P. \& Zalkin, H. (1992). Cloning and sequence of Bacillus subtilis purA and guaA, involved in the conversion of IMP to AMP and GMP. J Bacteriol 174, 1883-1890.

Marra, D. \& Scott, J. R. (1999). Regulation of excision of the conjugative transposon Tn916. Mol Microbiol 31, 609-621.

Martin, P., Trieu-Cuot, P. \& Courvalin, P. (1986). Nucleotide sequence of the tet $M$ tetracycline resistance determinant of the streptococcal conjugative shuttle transposon Tn1545. Nucleic Acids Res 14, 7047-7058.

Mullany, P., Wilks, M., Lamb, I., Clayton, C., Wren, B. \& Tabaqchali, S. (1990). Genetic analysis of a tetracycline resistance determinant from Clostridium difficile and its conjugal transfer to and from Bacillus subtilis. J Gen Microbiol 136, 1343-1349.

Mullany, P., Wilks, M. \& Tabaqchali, S. (1991). Transfer of Tn916 and Tn916 $\mathrm{E}$ into Clostridium difficile: demonstration of a hotspot for these elements in the C. difficile genome. FEMS Microbiol Lett 79, 191-194.

Mullany, P., Pallen, M., Wilks, M. \& Tabaqchali, S. (1996). A Group II intron in a conjugative transposon from the Grampositive bacterium, Clostridium difficile. Gene 174, 145-150.

Perreten, V., Schwarz, F., Cresta, L., Boeglin, M., Dasen, G. \& Teuber, M. (1997). Antibiotic resistance spread in food. Nature 389, 801-802. 
Poyart-Salmeron, C., Trieu-Cuot, P., Carlier, C. \& Courvalin, P. (1990). The integration-excision system of the conjugative transposon $\operatorname{Tn} 1545$ is structurally and functionally related to those of the lamboid phages. Mol Microbiol 4, 1513-1521.

Rice, L. B. (1998). Tn916 family of conjugative transposons and dissemination of antimicrobial resistance determinants. Antimicrob Agents Chemother 42, 1871-1877.

Rood, J. I. (1983). Transferable tetracycline resistance in Clostridium perfringens strains of porcine origin. Can J Microbiol 29, 1241-1246.

Rood, J. I., Maher, E. A., Somers, E. B., Campos, E. \& Duncan, C. L. (1978a). Isolation and characterization of multiply antibioticresistant Clostridium perfringens strains from porcine faeces. Antimicrob Agents Chemother 13, 871-880.

Rood, J. I., Scott, V. N. \& Duncan, C. L. (1978b). Identification of a transferable tetracycline resistance plasmid (pCW3) from Clostridium perfringens. Plasmid 1, 563-570.

Rudy, C. K., Scott, J. R. \& Churchward, G. (1997). DNA binding by the Xis protein of the conjugative transposon Tn916. Nucleic Acids Res 25, 4061-4066.

Sambrook, J., Fritsch, E. F. \& Maniatis, T. (1989). Molecular Cloning: a Laboratory Manual, 2nd edn. Cold Spring Harbor, NY: Cold Spring Harbor Laboratory.

Scott, J. R. \& Churchward, G. G. (1995). Conjugative transposition. Annu Rev Microbiol 49, 367-397.

Senghas, E., Jones, J. M., Yamamoto, M., Gawron-Burke, C. \& Clewell, D. B. (1988). Genetic organization of the bacterial conjugative transposon Tn916. J Bacteriol 170, 245-249.

Sloan, J., Warner, T. A., Scott, P. T., Bannam, T. L., Berryman, D. I. \& Rood, J. I. (1992). Construction of a sequenced Clostridium perfringens-Escherichia coli shuttle plasmid. Plasmid 27, 207-219.

Su, Y. A., He, P. \& Clewell, D. B. (1992). Characterization of the tet $(\mathrm{M})$ determinant of Tn916: evidence for regulation by tran- scription attenuation. Antimicrob Agents Chemother 36, 769-778.

Swartley, J. S., McAllister, C. F., Hajjeh, R. A., Heinrich, D. W. \& Stephens, D. S. (1993). Deletions of Tn916-like transposons are implicated in tetM-mediated resistance in pathogenic Neisseria. Mol Microbiol 10, 299-310.

Teuber, M., Meile, L. \& Schwarz, F. (1999). Acquired antibiotic resistance in lactic acid bacteria from food. Antonie Leeuwenhoek 76, 115-137.

Thompson, J. D., Higgins, D. G. \& Gibson, T. J. (1994). ClustAL w : improving the sensitivity of progressive multiple sequence alignment through sequence weighting, position-specific gap penalties and weight matrix choice. Nucleic Acids Res 22, 4673-4680.

Voelker, L. L. \& Dybvig, K. (1998). Characterisation of the lysogenic bacteriophage MAV1 from Mycoplasma arthritidis. J Bacteriol 180, 5928-5931.

Wang, H. \& Mullany, P. (2000). The large resolvase TndX is required and sufficient for the integration and excision of derivatives of the novel conjugative transposon Tn5397. J Bacteriol 182, 6577-6583.

Wang, H., Roberts, A. P., Lyras, D., Rood, J. I., Wilks, M. \& Mullany, P. (2000a). Characterisation of the ends and target sites of the novel conjugative transposon Tn5397 from Clostridium difficile: demonstration that excision and circularisation is mediated by TndX, a member of the large resolvase family. $J$ Bacteriol 182, 3775-3783.

Wang, H., Roberts, A. \& Mullany, P. (2000b). DNA sequence of the insertional hot-spot of Tn916 in the Clostridium difficile genome and discovery of a Tn916-like element in an environmental isolate integrated in the same hot spot. FEMS Microbiol Lett 192, 15-20.

Received 4 January 2001; revised 2 February 2001; accepted 5 February 2001. 